\title{
Proton therapy for patients with esophageal cancer: History, characteristics, clinical outcome and future direction of proton beam therapy
}

\author{
Masataka Karube, Hidetsugu Nakayama* \\ Department of Radiation Oncology, National Center for Global Health and Medicine, Tokyo, Japan.
}

\begin{abstract}
After the second war, Wilson who participated in development of the atomic bomb in Los Alamos studied peaceful use of atomic energy and proposed a property of proton beam that has potential to treat cancer. According to his proposal, the first patient was treated with proton beam therapy at the University of California Berkley in 1954. The first series of proton beam therapy for patients with esophageal cancer was reported from Japan in 1993. After that many proton facilities in Japan reported the clinical outcome of patients with esophageal cancer. Many dosimetric and clinical studies showed proton beam therapy for esophageal cancer was less toxic than photon beam therapy, however there is a paucity of randomized trials and evidence that proton beam therapy has clearly superior survival compared to photon therapy. Only one randomized trial has been conducted to study less toxicity for proton beam compared with intensity modulated radiotherapy (IMRT), which was stopped early because toxicities of IMRT were higher. A phase III study comparing overall survival between proton beam therapy and IMRT is now activated. A cost reduction for proton therapy is necessary to facilitate patient care and establishment of clinical evidence.
\end{abstract}

Keywords: proton beam therapy, esophageal cancer, particle beam therapy

\section{Introduction}

Proton beam therapy provides superior distribution of a high dose to tumors and low dose to normal tissue compared with photo beam (1). In the beginning of proton therapy for esophageal cancer, Japanese researchers played the main role for clinical application of proton beam therapy. Among various types of charged particles, now proton beam is the most widely used for esophageal cancer in the world.

In this article, we review dosimetric analysis of a proton plan for clinical results of reduced toxicity and survival, and discuss future directions of proton beam therapy.

\section{History of proton beam therapy}

Proton beams have a very rapid energy fall in the deep penetration site, which is known as the Bragg peak. This phenomenon was first reported by Sir William Henry Bragg in 1904 (2). Robert Wilson noted in 1948, using the Bragg peak, that a proton beam achieves desirable dose coverage of tumor volume and a therapeutic advantage for cancer compared with a photon beam (3). A dose distribution of protons is steep near the tumor and rapidly falls off behind the tumor (Figure 1). In
1954, the first proton therapy on humans was done for pituitary metastasis disseminated from breast cancer at the University of California Berkeley (4). After that proton therapy began at Uppsala, Sweden, Cambridge, United States of America and so on. In 1974, Suit et al. initiated studies of fractionated proton beam therapy for chordoma and chondrosarcoma at Harvard University (1). First proton therapy for uveal melanoma was also done at Harvard University (5). From their achievement, standard therapy for chordoma near the skull base and uveal melanoma even now use proton beam therapy. The largest number of patients with these cancers, in the world, are treated by proton beam. Contrary to this proton beam therapy is little used for thoracic, abdominal and pelvic cancer in 1900's.

In 1993 from Japan, Tsujii et al. reported results of proton beam therapy for these tumors included esophageal cancer at the University of Tsukuba (6). Nineteen patients with esophageal cancer were treated by proton beam. Seven patients underwent proton beam only with median dose of 78.5 Gy given in a median of 26 fractions. The other 12 patients were treated with photon proton with proton beam. The median combined total dose was $80.2 \mathrm{~Gy}$. The overall survival rates at 3 -years were $100 \%$ for Stage I, $60 \%$ for Stage II, and $50 \%$ for Stage III, respectively. They reported that the 


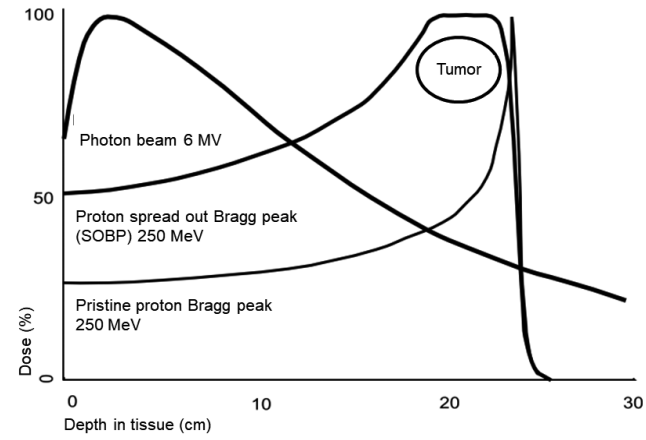

Figure 1. The shape of depth-dose curves for photon beam (6 $\mathrm{MV})$, pristine proton Bragg peak $(250 \mathrm{MeV})$ and scattered spread out Bragg peak (SOBP) proton beam. SOBP covers tumor well and energy is rapidly decreased behind the tumor. The energy of scattered SOBP proton beam is higher than pristine proton beam.

toxicity appeared to be minimum, even though the irradiated dose was higher than a conventional dose. To the best of our knowledge, this report is the first series of esophageal cancer that underwent proton beam therapy.

\section{Characteristics of proton beam therapy}

The proton beam is made from helium ions and accelerated using a cyclotron or synchrotrons to 230 $\mathrm{MeV}$ and more energy. The pristine proton beam generated from the accelerator is a narrow beam. For clinical use, in the two methods a "scattering" and "scanning" technique develop. The passive "scattering" method is spread out of the Bragg peak (SOBP) through the compensator (Figure 1). In this traditional proton beam therapy, the doses proximal to the tumor is similar to those of a photon. The new technique called active "scanning" develops, which is capable of being intensity modulated proton therapy (IMPT). IMPT is enabled to reduce the dose near the proximal site of the tumor. Hence, IMPT achieves an ideal dose distribution of protons, which consists of a low dose at the entrance, flat at the tumor and rapid fall off behind of the tumor.

The biological effect of radiation is different in organs and source of radiation. Comparing physical absorbed dose from different radiation sources, a coefficient named the relative biological effectiveness (RBE) is employed to compare the ratio of biological effectiveness of one type of ionizing radiation to another. Protons have a comparable similar biological effect as photon therapy. Many proton centers use RBE of protons as approximately 1.1. Contrarily Uppsala in Sweden use $\mathrm{RBE}$ as 1.0 as University of Tsukuba in Japan formerly used. The equation below is used to convert absorbed photon dose into proton absorbed dose.

Photon $(\mathrm{Gy})=\operatorname{RBE}(1.0$ or 1.1$) \times$ Proton $(\mathrm{Gy})$

The dose distributions of a proton beam are very sensitive to variation in tissue density through the beam pathway. The precise tissue density is necessary to evaluate by CT scanning and planned by advent of a treatment planning computer system. The control of organ motion or confirmation of tumor location by image guide are important to irradiate an accurate dose. The methods of breathing control, 4-dimentional (4D) planning CT, insertion of fiducial marker and image guide were developed for proton beam therapy and subsequently introduced to photon therapy.

\section{Dosimetric advantage of proton therapy}

Intensity modulated radiotherapy (IMRT) is widespread, which method delivers a photon beam more conformed to tumor and less to normal tissue, however an IMRT irradiated low dose of photons go around the tumor. Many showed a dosimetric advantage of even passive scattering from a much more active scanning proton beam compared with those of IMRT (7-13), because the dose of proton beam was a little behind the tumor. We review the dosimetry advantage, clinical outcome and future directions.

Esophagus is located at center of thorax and along the lung and heart. Lung is sensitive to radiation and has a risk of radiation pneumonitis. Heart is also at risk for pericarditis, cardiac effusion and myocardial infarction. To reduce such toxicities, lower dose of organ risk is ideal in a dosimetric plan. In modern radiotherapy, a CT scanning based dosimetric plan is calculated by a treatment planning computer.

Isacsson et al. described a passive proton beam plan reduced dose of the heart, lungs, spinal cord and kidneys compared with a photon beam plan in five patients with esophageal cancer (8). Zhang et al. showed a superior lung spring effect of passive proton plan to photon plan in 15 patients with distal esophageal cancer. They showed the maximum dose of spinal cord in 3-dimentional (3D) CT plan exceeded 5 Gy in that of 4-dimentional (4D) $\mathrm{CT}$ plan in proton beam therapy because of variations in stomach gas filling (13). This study warned precise planning is needed for a proton beam plan. Ling et al. also described the advantage of a passive proton plan compared to that of 3D conformal radiotherapy (CRT) and an IMRT plan in ten patients with esophageal cancer. They showed a proton plan consistently decreased the dose on the heart and lung compared with both 3D CRT and IMRT (9). Hirano et al. reported 27 patients with clinical stage III esophageal cancer compared among passive proton plan, 3D-CRT plan and IMRT plan in a dosimetric analysis. They showed proton plan reduced the dose of risk organs, especially lung and heart (7).

Shiraishi et al. demonstrated the heart dose of 727 patients with esophageal cancer comparing between proton and IMRT plans. The number of passive scanning proton therapy and IMPT were 237 and 13, respectively. They showed the proton beam plan resulted in significantly lower radiation exposure to the heart than IMRT plan. IMPT showed a significant decreased dose 
to heart compared with passive scanning proton (10). Zeng et al. demonstrated a comparison of beam direction of scattering proton and IMPT for 11 patients with esophageal cancer. Three beam directions, posterioranterior (PA), anterior-posterior/posterior-anterior (AP/PA) and posterior-anterior/left posterior oblique (PA/LPO) were compared. IMPT reduced the dose of proximal site of tumor compared with scattering proton beam. They showed proton therapy with a single PA IMPT was the most reduced dose for lung (12). These two studies showed the advantage of IMPT compared with scattering proton beam.

Warren et al. showed scanning proton plan reduced thoracic vertebrae dose compared with a bone marrow sparing volumetric modulated arc therapy (VMAT) plan in 21 patents with mid-esophageal cancer (11). They speculated a reduced dose of vertebral bone marrow by proton beam has potential to reduce acute toxicities in concurrent chemoradiotherapy for esophageal cancer.

\section{Reduced clinical toxicities of proton therapy}

There were several clinical reports, which described proton beam therapy reduced toxicities compared with photon therapy. Makishima et al. from University of Tsukuba showed an advantage of dose histogram for passive proton beam therapy and retrospectively compared adverse events $(n=24)$ with photon $(n=$ 13) beam. Radiation pneumonitis and cardiac effusion was significantly reduced using proton beam therapy (14). Wang et al. from MD Anderson Cancer Center reported they compared gastrointestinal and pulmonary complication among 444 patients treated with 3D-CRT, IMRT and passive proton beam therapy. The proton beam had lower complications than others, and the median length of hospital stay was significantly shorter with proton beam (15).

Fang et al. reported from MD Anderson Cancer Center, passive proton beam therapy had a low rate of lymphocytopenia during definitive chemoradiotherapy compared with IMRT. Patients underwent proton beam therapy $(n=110)$ and was matched by propensity score with patients treated with IMRT $(n=110)$. On multivariate analysis, proton beam therapy had a lower risk of Grade 4 rate of lymphocytopenia (hazard ratio $[\mathrm{HR}]=0.5, p=0.01$ ) than IMRT (16). Shiraishi et al. from MD Anderson Cancer Center, compared lymphocyte counts on esophageal cancer treated neoadjuvant chemoradiotherapy between passive proton beam therapy and IMRT. Patients' characteristics were matched by propensity score. One hundred thirty-six patients of each group were studied. Radiation dose was 50.4 Gy given in 28 fractions in each group. Grade 4 lymphopenia was significantly less in proton beam therapy compared with IMRT. Proton beam was significantly associated with a reduction in Grade 4 lymphopenia on multivariable analysis.
They concluded proton beam therapy prevented Grade 4 lymphopenia during chemoradiotherapy (17). Routman et al. from Mayo Clinic also reported scanning proton beam reduced Grade 4 lymphopenia during chemoradiotherapy. Seventy-nine and 65 patients were treated with photon and proton beam therapy, respectively. All patients received 41.4 - 50.4 Gy. On multi- and uni-variate analysis they showed proton beam therapy was significantly associated with reduction of Grade 4 lymphopenia (18). Lymphopenia is associated with survival of patients with esophageal cancer who underwent chemoradiotherapy (19). Lymphocytes are one of the most vulnerable organs to radiation. These results seem to show a dosimetric advantage of proton beam translated into clinical outcome.

Garant et al. from Mayo Clinic demonstrated proton beam therapy showed less decline in health-related quality of life (HRQOL) during chemoradiotherapy compared with photon beam therapy. One hundred eighty-nine patients were assessed using the functional assessment of cancer therapy-esophageal (FACT-E) before and after chemoradiotherapy. On multi- and univariate analysis proton beam was associated with less decline in FACT-E scores compared with photon beam (20).

\section{Clinical data of proton therapy for esophageal cancer}

Sugahara et al. from University of Tsukuba initially reported clinical results of esophageal cancer treated passive proton beam therapy (21) and afterwards Mizumoto et al. updated the initial report (22). The numbers of clinical stages I, II and III were $8(15.7 \%)$, $23(45.1 \%)$ and $20(39.2 \%)$ patients, respectively. Chemotherapy was not done. Thirty-three patients were treated using photon therapy with median dose of 46 Gy (range 7-60 Gy) followed by proton boost with median dose of 36 Gy (range 7-60 Gy). Total median dose of photon and proton beam was 80 Gy (range 70-90 Gy). Eighteen patients were treated using proton alone with median dose of 79 Gy (range 62-98 Gy). No patients had a treatment interruption due to hematological toxicity. One patient was discontinued because of aspiration pneumonia. Acute toxicity was relatively mild, and six patients had Grade 3 esophagitis. One patient died because of esophageal ulcer. The patients receiving $80 \mathrm{~Gy}$ and more had more frequent esophageal ulcer compared with less than 80 Gy. The 5-year overall survival and local control rates for all 51 patients were $21.1 \%$ and $38.0 \%$, respectively. The complete response (CR) rates were $100 \%$ for patients at the T1 or T2 stage, $77 \%$ for T3, and 38\% for T4, respectively. Thirty-three percent of patients had recurrence at the primary site. On uni- and multi-variate analysis, prognostic factors for overall survival were only for $\mathrm{T}$ stage and that for local control were not identified.

Ishikawa et al. reported concurrent chemotherapy 
with passive proton beam therapy for 40 patients from University of Tsukuba. The number of clinical stages I, II and III were 16 (40\%), 9 (22.5\%) and 15 (37.5\%) patients, respectively. The dose of 60 Gy was irradiated given in 30 fractions. When residual tumor was observed at 50 Gy by endoscopic examination, an additional dose of 4-10 Gy was boosted. Twenty-one patients had undergone the boost proton beam. Ten and nine patients had Grade 3 or 4 hematological and esophagitis toxicities, respectively. Late Grade 3 toxicities occurred only in two patients. Two patients with T3 disease had stricture of esophagus and ulcer with residual tumor in each. The-3 year overall survival was $70.4 \%$. The 2 -year overall survival and local control rates for all was $75.1 \%$ and $66.4 \%$, respectively. The clinical CR rates for stage I, II and III were $88 \%, 89 \%$ and $56 \%$, respectively. 56\% recurred at the primary site (23).

Zeng et al. from University of Washington described preliminary results of IMPT for 13 patients with esophageal cancer. All patients underwent neoadjuvant IMPT with a chemotherapy dose of 50.4 Gy given in 28 fractions followed by surgery. Tumor stage and histology were cT3-4 distal esophageal adenocarcinoma. Grade 4 and more toxicity had not occurred during IMPT. Twelve patients underwent surgery after IMPT except one patient because of progression of systemic disease. Of all 12 patients who underwent surgery, pathological $\mathrm{CR}$ was seen in $25 \%$ and $\mathrm{R} 0$ resection was achieved in all patients (12).

Lin et al. from MD Anderson Cancer Center reported the outcome of 62 patients with esophageal cancer who underwent passive proton beam therapy with dose of 50.4 Gy given in 28 fractions. The numbers of adenocarcinoma and squamous cell carcinoma were 47 (75.8\%) and $14(22.6 \%)$, respectively. Most patients were stage II - III disease (84\%). Thirty-three (53.2\%) and 29 (46.8\%) of patients underwent definitive radiotherapy and radiotherapy followed by surgery, respectively. The pathological CR rate was $28 \%$. Proton beam therapy was well tolerated. The rate of Grade 2-3 pneumonitis was $3.2 \%$. The 3 -year overall survival and local control rates for definitive radiotherapy were $51.7 \%$ and $56.5 \%$, respectively (24).

Takeda et al. from Southern Tohoku Proton Center reported the results of 47 patients with esophageal cancer treated with photon beam followed by passive proton boost with chemotherapy. The doses of photon and proton were 36 Gy given in 20 fractions and 33-39.6 Gy given in 15-18 fractions, respectively. The number of stages I, II and III were 10 (21.3\%), 12 (25.5\%) and $25(53.1 \%)$ patients, respectively. None had Grade 4 and more toxicity. One patient $(2.1 \%)$ had Grade 3 pneumonitis. The 3 -year overall survival and local control rates were $59.2 \%$, and $69.8 \%$, respectively (25).

Ono et al. reported clinical results of 202 patients with esophageal cancer who underwent definitive proton beam therapy from a multicenter in Japan. Seventy- two (35.6\%), 30 (14.9\%), $52(25.7 \%)$ and $48(23.8 \%)$ patients had clinical stage I, II, III and IV disease, respectively. The median total dose was $87.2 \mathrm{~Gy}$. The 3and 5-year overall survival rates were $66.7 \%$ and $56.3 \%$, respectively. The 5-year overall survival rates for stages I, II, III, and IV were $79.3 \%, 66.3 \%, 43.2 \%$, and $28.3 \%$, respectively. The 3- and 5-year local control rates for all were 70.2 and $64.4 \%$, respectively. None had Grade 4 or more toxicities. There was one patient who had Grade 3 pericardial effusion and pneumonia (26).

Table 1 shows a summary of clinical outcomes. Interpreting outcomes are difficult due to various doses, stage and type of histology, however a dose over 60 Gy with chemotherapy appear to be superior for overall survival and local control rate for historical photon beam therapy (27).

\section{Comparison of clinical outcomes between proton and photon beam therapy}

Table 2 shows a comparison of clinical outcomes between proton and photon beam therapy. Xi et al. reported survival benefit of passive proton beam therapy retrospectively compared with IMRT from MD Anderson Cancer Center. They compared 343 patients with esophageal cancer who received definitive chemoradiotherapy with proton beam therapy $(n=132)$ or IMRT $(n=211)$. The dose was 50.4 Gy given in 28 fractions and the median dose was both 50.4 Gy for the IMRT (41.4-66 Gy) and proton beam therapy (45-63 Gy). The number of clinical stage I/II and III were 117 (34.1\%) and $226(65.9 \%)$, respectively. Proton beam therapy had significantly better overall survival $(p=0.011)$ compared with IMRT. Local control rate was marginal $(p=0.075)$. Treatment related toxicities were not significant between the two groups. 5-year overall survival for patients with stage III disease was significantly better for proton beam (34.6\%) than IMRT (25\%) (28).

Lin et al. prospectively studied total toxicity burden and progression-free survival between proton beam therapy and IMRT in multicenters of the United States of America. Six (5.6\%), 41 (38.3\%) and 60 (56.1\%) had clinical stage I, II and III, respectively. Ninety-five $(88.8 \%)$ and $89(83.2 \%)$ patients had adenocarcinoma and at a lower location of esophagus, respectively. The dose of proton beam and IMRT was 50.4 Gy given in 28 fractions. One hundred forty-five patients were randomly assigned and 107 patients were evaluated because of an early stopping rule at the interim analysis. The total toxicity burden was 2.3 times higher for IMRT than proton beam therapy. The 3-year progression-free survival (50.8\% vs. 51.2\%) and 3-year overall survival rates $(44.5 \%$ vs. $44.5 \%)$ were similar (29). Two studies proved dosimetric advantages of proton beam compared with IMRT translated to improved clinical outcomes. These suggest decreased toxicity of proton beam therapy for esophageal cancer may induce prolonged survival 


and possible dose escalation without increasing toxicity.

\section{Future directions}

The standard dose of concurrent chemoradiotherapy for locally advanced esophageal cancer is 50.4 Gy given in 28 fractions, which was determined by the radiation therapy oncology group (RTOG) 94-04/INT 0123 trial (27). A higher dose of 64.8 Gy given in 36 fractions was expected to improve overall survival, however overall survival of a higher dose group at 2-years was lower than the lower dose of 50.4 Gy given in 28 fractions. Treatment-related deaths were higher in the high dose group than the lower group, which affected survival of the high dose group (27). If treatment-related effects could be reduced by proton beam, overall survival could possibly improve.

Several dose escalating studies were reported using photon beam. Welsh et al. reported the results of a phase I/II trial from MD Anderson Center. Foyty-four patients underwent chemoradiotherapy with a simultaneous integrated boost (SIB) of 58.8 to $63 \mathrm{~Gy}$. Local control rate at 1 -year was $69.9 \%$. They concluded that doseescalation may improve local control (30). Yu et al. reported 45 patients underwent 63 Gy with SIB. Local control rates were $83.3 \%$ at 1 -year and $67.5 \%$ at 3 -years (31). Finally, Luo et al. reported the result of metaanalysis for the effect of modern high-dose compared with standard dose photon therapy. They showed high dose improved overall survival $(\mathrm{HR}=0.78, p<0.001)$ and concluded high dose based on modern radiotherapy appears to improve overall survival (32).

Mizumoto et al. reported results of concomitant proton boost combined with photon therapy. Nineteen patients underwent this hyper-fractionated radiotherapy. Total irradiated dose ranged from 74 Gy to 80 Gy. Seventeen $(89 \%)$ patients achieved CR. The $1-$ and 5 -year local control rates for all 19 patients were $93.8 \%$ and $84.4 \%$, respectively (33). It is necessary for radiation oncology to prove improvement of survival not only reduced toxicity. In 2008, Suit et al. reported data of much value to radiation oncology to determine the clinical consequence of changes in dose, and dose fractionation (34). Based on a photon dose escalation study and results of proton therapy, dose escalation may improve survival for esophageal cancer. A phase I study of dose escalation proton beam therapy has been activated at the University of Pennsylvania.

A phase III randomized trial (NRG-GI006) comparing proton beam therapy versus IMRT has been started (35). The primary endpoint is non-inferior overall survival with proton beam therapy compared with IMRT and less than Grade 3 and more cardiopulmonary toxicity. The dose of 50.4 Gy given in 28 fractions with chemotherapy is irradiated in esophageal cancer in two groups. An active scanning proton center is increased in the United States of America, so active scanning expects much lower toxicities than passive scattering in this trial.

Some passive proton beam center, with a wide area $(25 \times 25 \mathrm{~cm})$ is difficult to irradiate, so proton beam is used as a boost after photon therapy. Many clinical reports from Japan included combined photon and passive scattering proton beam therapy. Passive scattering proton has a high dose in front of the tumor and does not irradiate the entire esophagus. In the dosimetric plan analysis, a scanning proton plan was superior to a scattering plan. Scanning proton beam therapy may provide the original property of proton beam in the clinic.

High cost is a great criticism for proton beam therapy. Fortunately, cost is gradually deceasing, however, large space and a high running cost is needed to generate high energy proton beam. In Japan, the cost of esophageal cancer undergoing proton beam therapy is three times higher than IMRT. This is one of the reasons randomized trials are lacking. Laser accelerated proton beam, which has a unique niche is now under development (36). Laser accelerated protons do not use a synchrotron or cyclotron, so space and running cost is low. Laser accelerated protons are relatively low energy with wide energy, and low reproducibility. After these problems are resolved, laser accelerated proton beam therapy may spread widely just as the linear accelerator replaced Cobalt-60 $\left({ }^{60} \mathrm{Co}\right)$. If proton beam therapy has low cost, many patients will receive proton beam therapy and clinical trials will be enhanced.

\section{Funding: None.}

Conflict of Interest: The authors have no conflicts of interest to disclose.

\section{References}

1. Suit HD, Goitein M, Munzenrider J, Verhey L, Davis KR, Koehler A, Linggood R, Ojemann RG. Definitive radiation therapy for chordoma and chondrosarcoma of base of skull and cervical spine. J Neurosurg. 1982; 56:377-385.

2. Bragg WH, Kleeman R. LXXIV. On the ionization curves for radium. The London, Edinburgh, and Dublin Philosophical Magazine and Journal of Science. 1904; 8:726-738.

3. Wilson RR. Radiological use of fast protons. Radiology. 1946; 47:487-491.

4. Lawrence JH, Tobias CA, Born JL, Mc Combsrk RK, Roberts JE, Anger HO, Low-Beer BV, Huggins CB. Pituitary irradiation with high-energy proton beams: a preliminary report. Cancer Res. 1958; 18:121-134.

5. Gragoudas ES, Goitein M, Koehler AM, Verhey L, Tepper J, Suit HD, Brockhurst R, Constable IJ. Proton irradiation of small choroidal malignant melanomas. Am J Ophthalmol. 1977; 83:665-673.

6. Tsujii H, Tsuji H, Inada T, Maruhashi A, Hayakawa Y, Takada Y, Tada J, Fukumoto S, Tatuzaki H, Ohara K, Kitagawa T. Clinical results of fractionated proton therapy. Int J Radiat Oncol Biol Phys. 1993; 25:49-60. 
7. Hirano $\mathrm{Y}$, Onozawa M, Hojo H, Motegi A, Zenda S, Hotta K, Moriya S, Tachibana H, Nakamura N, Kojima T, Akimoto T. Dosimetric comparison between proton beam therapy and photon radiation therapy for locally advanced esophageal squamous cell carcinoma. Radiat Oncol. 2018; 13:23.

8. Isacsson U, Lennernas B, Grusell E, Jung B, Montelius A, Glimelius B. Comparative treatment planning between proton and $\mathrm{x}$-ray therapy in esophageal cancer. Int $\mathrm{J}$ Radiat Oncol Biol Phys. 1998; 41:441-450.

9. Ling TC, Slater JM, Nookala P, Mifflin R, Grove R, Ly AM, Patyal B, Slater JD, Yang GY. Analysis of Intensity-Modulated Radiation Therapy (IMRT), Proton and 3D Conformal Radiotherapy (3D-CRT) for Reducing Perioperative Cardiopulmonary Complications in Esophageal Cancer Patients. Cancers (Basel). 2014; 6:2356-2368.

10. Shiraishi Y, Xu C, Yang J, Komaki R, Lin SH. Dosimetric comparison to the heart and cardiac substructure in a large cohort of esophageal cancer patients treated with proton beam therapy or intensity-modulated radiation therapy. Radiother Oncol. 2017; 125:48-54.

11. Warren S, Hurt CN, Crosby T, Partridge M, Hawkins MA. Potential of Proton Therapy to Reduce Acute Hematologic Toxicity in Concurrent Chemoradiation Therapy for Esophageal Cancer. Int J Radiat Oncol Biol Phys. 2017; 99:729-737.

12. Zeng YC, Vyas S, Dang Q, Schultz L, Bowen SR, Shankaran V, Farjah F, Oelschlager BK, Apisarnthanarax $\mathrm{S}$, Zeng J. Proton therapy posterior beam approach with pencil beam scanning for esophageal cancer : Clinical outcome, dosimetry, and feasibility. Strahlenther Onkol. 2016; 192:913-921.

13. Zhang X, Zhao KL, Guerrero TM, McGuire SE, Yaremko B, Komaki R, Cox JD, Hui Z, Li Y, Newhauser WD, Mohan R, Liao Z. Four-dimensional computed tomography-based treatment planning for intensitymodulated radiation therapy and proton therapy for distal esophageal cancer. Int J Radiat Oncol Biol Phys. 2008; 72:278-287.

14. Makishima H, Ishikawa $H$, Terunuma $T$, Hashimoto $T$, Yamanashi K, Sekiguchi T, Mizumoto M, Okumura T, Sakae T, Sakurai H. Comparison of adverse effects of proton and X-ray chemoradiotherapy for esophageal cancer using an adaptive dose-volume histogram analysis. J Radiat Res. 2015; 56:568-576.

15. Wang J, Wei C, Tucker SL, Myles B, Palmer M, Hofstetter WL, Swisher SG, Ajani JA, Cox JD, Komaki R, Liao Z, Lin SH. Predictors of postoperative complications after trimodality therapy for esophageal cancer. Int $\mathrm{J}$ Radiat Oncol Biol Phys. 2013; 86:885-891.

16. Fang P, Shiraishi Y, Verma V, Jiang W, Song J, Hobbs BP, Lin SH. Lymphocyte-sparing effect of proton therapy in patients with esophageal cancer treated with definitive chemoradiation. Int J Part Ther. 2018; 4:23-32.

17. Shiraishi Y, Fang P, Xu C, et al. Severe lymphopenia during neoadjuvant chemoradiation for esophageal cancer: A propensity matched analysis of the relative risk of proton versus photon-based radiation therapy. Radiother Oncol. 2018; 128:154-160.

18. Routman DM, Garant A, Lester SC, Day CN, Harmsen WS, Sanheuza CT, Yoon HH, Neben-Wittich MA, Martenson JA, Haddock MG, Hallemeier CL, Merrell KW. A comparison of grade 4 lymphopenia with proton versus photon radiation therapy for esophageal cancer.
Adv Radiat Oncol. 2019; 4:63-69.

19. Yoo EJ, Park JC, Kim EH, Park CH, Shim CN, Lee HJ, Chung HS, Lee H, Shin SK, Lee SK, Lee CG, Lee YC. Prognostic value of neutrophil-to-lymphocyte ratio in patients treated with concurrent chemoradiotherapy for locally advanced oesophageal cancer. Dig Liver Dis. 2014; 46:846-853.

20. Garant A, Whitaker TJ, Spears GM, et al. A comparison of patient-reported health-related quality of life during proton versus photon chemoradiation therapy for esophageal cancer. Pract Radiat Oncol. 2019; 9:410-417.

21. Sugahara S, Tokuuye K, Okumura T, Nakahara A, Saida Y, Kagei K, Ohara K, Hata M, Igaki H, Akine Y. Clinical results of proton beam therapy for cancer of the esophagus. Int J Radiat Oncol Biol Phys. 2005; 61:76-84.

22. Mizumoto M, Sugahara S, Nakayama H, Hashii H, Nakahara A, Terashima H, Okumura T, Tsuboi K, Tokuuye K, Sakurai H. Clinical results of proton-beam therapy for locoregionally advanced esophageal cancer. Strahlenther Onkol. 2010; 186:482-488.

23. Ishikawa H, Hashimoto T, Moriwaki T, Hyodo I, Hisakura K, Terashima H, Ohkohchi N, Ohno T, Makishima H, Mizumoto M, Ohnishi K, Okumura T, Sakurai H. Proton beam therapy combined with concurrent chemotherapy for esophageal cancer. Anticancer Res. 2015; 35:1757-1762.

24. Lin SH, Komaki R, Liao Z, Wei C, Myles B, Guo X, Palmer M, Mohan R, Swisher SG, Hofstetter WL, Ajani JA, Cox JD. Proton beam therapy and concurrent chemotherapy for esophageal cancer. Int J Radiat Oncol Biol Phys. 2012; 83:e345-351.

25. Takada A, Nakamura T, Takayama K, et al. Preliminary treatment results of proton beam therapy with chemoradiotherapy for stage I-III esophageal cancer. Cancer Med. 2016; 5:506-515.

26. Ono $\mathrm{T}$, Wada $\mathrm{H}$, Ishikawa $\mathrm{H}$, Tamamura $\mathrm{H}$, Tokumaru S. Clinical results of proton beam therapy for esophageal cancer: multicenter retrospective study in Japan. Cancers (Basel). 2019; 11:993.

27. Minsky BD, Pajak TF, Ginsberg RJ, Pisansky TM, Martenson J, Komaki R, Okawara G, Rosenthal SA, Kelsen DP. INT 0123 (Radiation Therapy Oncology Group 94-05) phase III trial of combined-modality therapy for esophageal cancer: high-dose versus standard-dose radiation therapy. J Clin Oncol. 2002; 20:1167-1174.

28. Xi M, Xu C, Liao Z, et al. Comparative outcomes after definitive chemoradiotherapy using proton beam therapy versus intensity modulated radiation therapy for esophageal cancer: a retrospective, single-institutional analysis. Int J Radiat Oncol Biol Phys. 2017; 99:667-676.

29. Lin SH, Hobbs BP, Verma V, et al. Randomized phase IIB trial of proton beam therapy versus intensity-modulated radiation therapy for locally advanced esophageal cancer. J Clin Oncol. 2020; 38:1569-1579.

30. Welsh JW, Seyedin SN, Allen PK, et al. Local control and toxicity of a simultaneous integrated boost for dose escalation in locally advanced esophageal cancer: interim results from a prospective phase I/II Trial. J Thorac Oncol. 2017; 12:375-382.

31. Yu WW, Zhu ZF, Fu XL, Zhao KL, Mao JF, Wu KL, Yang HJ, Fan M, Zhao S, Welsh J. Simultaneous integrated boost intensity-modulated radiotherapy in esophageal carcinoma: early results of a phase II study. Strahlenther Onkol. 2014; 190:979-986.

32. Luo HS, Huang HC, Lin LX. Effect of modern high-dose versus standard-dose radiation in definitive concurrent 
chemo-radiotherapy on outcome of esophageal squamous cell cancer: a meta-analysis. Radiat Oncol. 2019; 14:178.

33. Mizumoto M, Sugahara S, Okumura T, Hashimoto T, Oshiro Y, Fukumitsu N, Nakahara A, Terashima H, Tsuboi K, Sakurai H. Hyperfractionated concomitant boost proton beam therapy for esophageal carcinoma. Int $\mathrm{J}$ Radiat Oncol Biol Phys. 2011; 81:e601-606.

34. Suit H, Kooy H, Trofimov A, Farr J, Munzenrider J, DeLaney T, Loeffler J, Clasie B, Safai S, Paganetti H. Should positive phase III clinical trial data be required before proton beam therapy is more widely adopted? No. Radiother Oncol. 2008; 86:148-153.

35. NRG-GI006 Phase III randomized trial of proton beam therapy (PBT) versus intensity modulated photon radiotherapy (IMRT) for the treatment of esophageal cancer. https://www.nrgoncology.org/Clinical-Trials/ Protocol/nrg-gi006 (accessed January 19, 2021)
36. Karsch L, Beyreuther E, Enghardt W, Gotz M, Masood U, Schramm U, Zeil K, Pawelke J. Towards ion beam therapy based on laser plasma accelerators. Acta Oncol. 2017; 56:1359-1366.

Received September 24, 2020; Revised January 20, 2021; Accepted January 28, 2021.

Released online in J-STAGE as advance publication February $11,2021$.

\section{*Address correspondence to:}

Hidetsugu Nakayama, Department of Radiation Oncology, National Center for Global Health and Medicine, 1-21-1 Toyama, Shinjyuku-ku, Tokyo 162-8655, Japan.

E-mail: hnakayama@hosp.ncgm.go.jp 